Rev Soc Esp Dolor

2014; 21(2): 118-119

\section{Tratamiento de espasmos musculares en el paciente con ataxia de Friedreich con bomba de infusión intratecal de baclofeno: a propósito de un caso}

\section{Sr. Director:}

La ataxia de Friedreich (AF), descrita por Nikolaus Friedreich en 1863, es un síndrome atáxico degenerativo hereditario con un patrón de herencia autosómica recesiva debida a la expansión del triplete GAA del primer intrón del gen FXN en el cromosoma 9q13, que provoca una importante disminución de la frataxina, una proteína de la matriz mitocondrial quelante del hierro que deriva en una temprana y progresiva neurodegeneración del cerebelo y los ganglios espinales dorsales. Es el síndrome atáxico hereditario más frecuente, siendo su prevalencia en Europa entre 1:20.000 y 1:125.000. El fenotipo clínico clásico incluye alteraciones del equilibrio y coordinación, debilidad principalmente en las extremidades inferiores, pérdida de sensibilidad, arreflexia, alteraciones de la marcha, disartria, disfagia, alteraciones en el movimiento ocular, escoliosis, deformidades de pie, cardiomiopatía y diabetes (1) y suele debutar en la pubertad. El diagnóstico fundamentalmente es clínico, aunque en la actualidad puede hacerse diagnóstico precoz con estudios genéticos.

Presentamos el caso de un varón de 49 años. A los 11 años de edad comenzó un cuadro de alteraciones al caminar, inestabilidad y caídas frecuentes, siendo diagnosticado con 15 años de edad de AF. El paciente no presenta déficit cognitivo y debido a su ataxia cerebelosa se encuentra en situación de paraplejia, presenta disartria y es dependiente para aseo y vestido. Tiene una leve espasticidad de grado 1 en la escala de Asworth y espasmos musculares frecuentes, principalmente en decúbito supino, que han presentado un empeoramiento en los últimos 7 meses, grado 3 en la escala de Penn, hasta imposibilitar el descanso nocturno. Estos espasmos se trataron inicialmente con baclofeno vía oral, con buena respuesta, pero la dosis necesaria actual $(75 \mathrm{mg}$ repartidos en 3 dosis) producía una somnolencia diurna excesiva. Nuestro paciente presenta otras afectaciones orgánicas propias de la AF como miocardiopatía dilatada y diabetes mellitus. Como antecedentes quirúrgicos destaca la realización de 16 fibrotomías percutáneas tipo fibrotomía gradual de Ulzibat a los 37 años en las extremidades inferiores.
Se realizó el estudio preoperatorio con electrocardiograma y analítica con coagulación, sin alteraciones importantes. Se había sustituido acenocumarol por enoxaparina y se pautó profilaxis antibiótica con cefazolina según los protocolos de nuestro centro.

Según la técnica quirúrgica habitual, bajo sedación profunda y anestesia local, se prodece a la implantación de un catéter intratecal InDura 1P modelo 8709SC de Medtronic (Medtronic, EE.UU.) y una bomba de infusión implantable de flujo constante Tricumed IP2000V (Tricumed Medizintechnik $\mathrm{GmbH}$, Alemania), sin incidencias intraquirúrgicas.

El postoperatorio transcurre sin incidencias y se procede al inicio de infusión continua intradural de baclofeno a $50 \mu \mathrm{g} / \mathrm{día}$. En las primeras 24 horas el paciente refiere mejoría de los espasmos musculares y del descanso nocturno, por lo que es dado de alta. En las revisiones sucesivas persiste la mejoría referida por el paciente, objetivando disminución de la frecuencia de espasmos a grado 2 de la escala de Penn y se optimiza dosis, actualmente a $75 \mu \mathrm{g} /$ día.

El baclofeno es un relajante muscular de acción central que deprime la transmisión refleja monosináptica y polisináptica en la médula espinal por estimulación de receptores GABAB, sin afectar a la transmisión neuromuscular. El tratamiento con baclofeno intratecal está indicado en la espasticidad crónica grave de origen espinal o cerebral, destacando su utilización en lesiones espinales, esclerosis múltiple y parálisis cerebrales, que no respondan a antiespásticos por vía oral y/o que sufran efectos secundarios inaceptables con dosis orales eficaces y tengan un flujo normal de líquido cefalorraquídeo. El empleo de baclofeno intratecal para la espasticidad de origen supraespinal es una terapia de eficacia contrastada en la literatura médica $(2,3)$. Nuestro paciente había experimentado buena respuesta a baclofeno vía oral, pero con la progresión de la enfermedad la dosis necesaria provocaba somnolencia excesiva, por lo que lo consideramos candidato a la bomba de infusión de baclofeno intratecal.

En la bibliografía consultada se encuentra una única referencia específica al empleo de baclofeno intratecal para el tratamiento de los espasmos musculares en la AF, con mejoría sintomática del caso clínico presentado (4).

F. Curt Nuño, M.A. Pérez Díaz, M. López Rodríguez, M. González Cabano, C. Rodríguez Bello y A. Camba Rodríguez 
Unidad del Dolor. Servicio de Anestesia, Reanimación y Terapéutica del Dolor. Complejo Hospitalario Universitario de Ferrol. Ferrol, La Coruña

\section{Bibliografía}

1. Parkinson MH, Boesch S, Nachbauer W, Mariotti C, Giunti P. Clinical features of Friedreich's ataxia: classical and atypical phenotypes. J Neurochem 2013;126(Supl. 1):103-17.
2. Bensmail D, Quera Salva MA, Roche N, Benyahia S, Bohic $M$, Denys P, et al. Effect of intrathecal baclofen on sleep and respiratory function in patients with spasticity. Neurology 2006;67:1432-6.

3. Stokic DS, Yablon SA, Hayes A. Comparison of Clinical and Neurophysiologic Responses to Intrathecal Baclofen Bolus Administration in Moderate-to- Severe Spasticity After Acquired Brain Injury. Arch Phys Med Rehabil 2005;86:1801-6.

4. Ben Smail D, Jacq C, Denys P, Bussel B. Intrathecal baclofen in the treatment of painful, disabling spasms in Friedreich's ataxia. Mov Disord 2005;20:758-9. 